\title{
Selective Addition of Palladium on the Rim of Open-cage Fullerenes to Form Mononuclear and Dinuclear
}

\section{Complexes}

\author{
Hao Zhang, ${ }^{a}$ Zishuo Zhou, ${ }^{\mathrm{a}}$ Le Yang, ${ }^{\mathrm{b}}$ Jie Su, ${ }^{\mathrm{a} *}$ Peng Jin, ${ }^{\mathrm{b}}{ }$ Liangbing Gan ${ }^{\mathrm{a}, \mathrm{c}_{*}}$ \\ ${ }^{\text {a }}$ Beijing National Laboratory for Molecular Sciences, Key Laboratory of Bioorganic Chemistry and \\ Molecular Engineering of the Ministry of Education, College of Chemistry and Molecular Engineering \\ Peking University, Beijing 100871, China; ${ }^{\mathrm{b}}$ School of Materials Science and Engineering, Hebei \\ University of Technology, Tianjin 300130, China; ${ }^{\mathrm{c}}$ State Key Laboratory of Organometallic Chemistry \\ Shanghai Institute of Organic Chemistry, Shanghai 200032, China.
}

All reagents were used as received. All solvents were used as received. The reactions were carried out under atmosphere condition. The NMR spectra were obtained at $25{ }^{\circ} \mathrm{C}$ with $400 \mathrm{MHz}$ spectrometers $\left({ }^{1} \mathrm{H}\right.$ NMR spectra for the same compound were obtained with different spectrometers and different solvents in some cases). Chemical shifts are given in ppm relative to CDCl3. ESI-FT-ICR-HRMS spectra were recorded in positive mode. Chromatographic purifications were carried out with silica gel of mesh 200-300.

Known compounds were characterized by comparison of their ${ }^{1} \mathrm{H}$ NMR data with literature data as cited.

Note: common impurities such as residue solvent toluene and grease from silica gel are sometimes impossible to be removed from the fullerene derivatives by routine flash column chromatography. The diffusion-precipitation solvents are $\mathrm{CHCl}_{3}$ /hexane or $\mathrm{CS}_{2} / \mathrm{CH}_{3} \mathrm{CN}$. 


\section{Reaction Scheme S1}
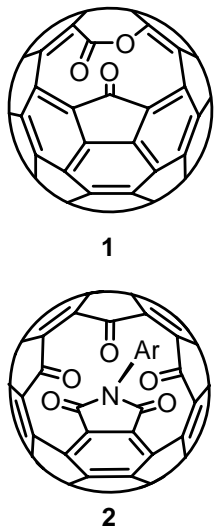

$\mathrm{Pd}(\mathrm{OAc})_{2}, \mathrm{PPh}_{3}$ $\mathrm{CHCl}_{3}$, r. t. mixture

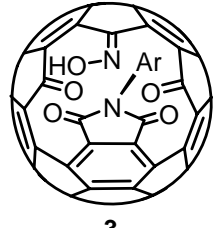

$\underset{\text { Tol, r. t. }}{\stackrel{\mathrm{Pd}\left(\mathrm{PPh}_{3}\right)_{4}}{\longrightarrow}}$ $60.4 \%$

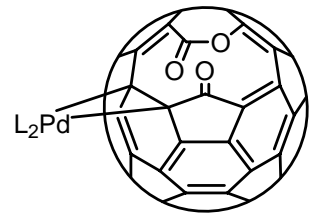

4

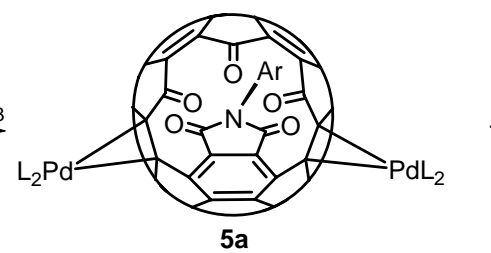

$5 a$

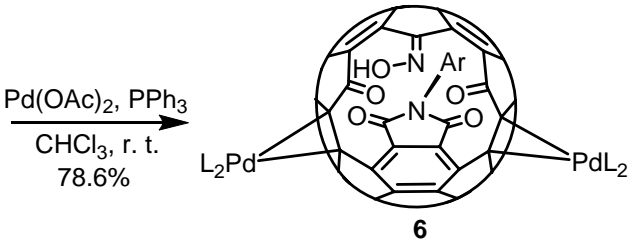

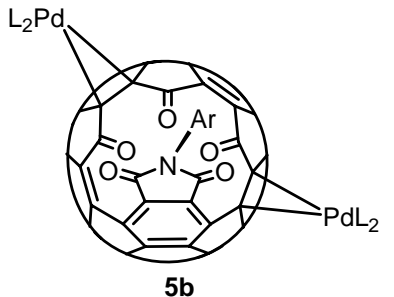

$L=\mathrm{PPh}_{3} \quad \mathrm{Ar}=$

Figure S1 ${ }^{1} \mathrm{H}$ NMR of Compounds $\mathrm{H}_{2} \mathrm{O} @ 5 \mathrm{a} / \mathrm{b}$ in $\mathrm{CDCl}_{3}$

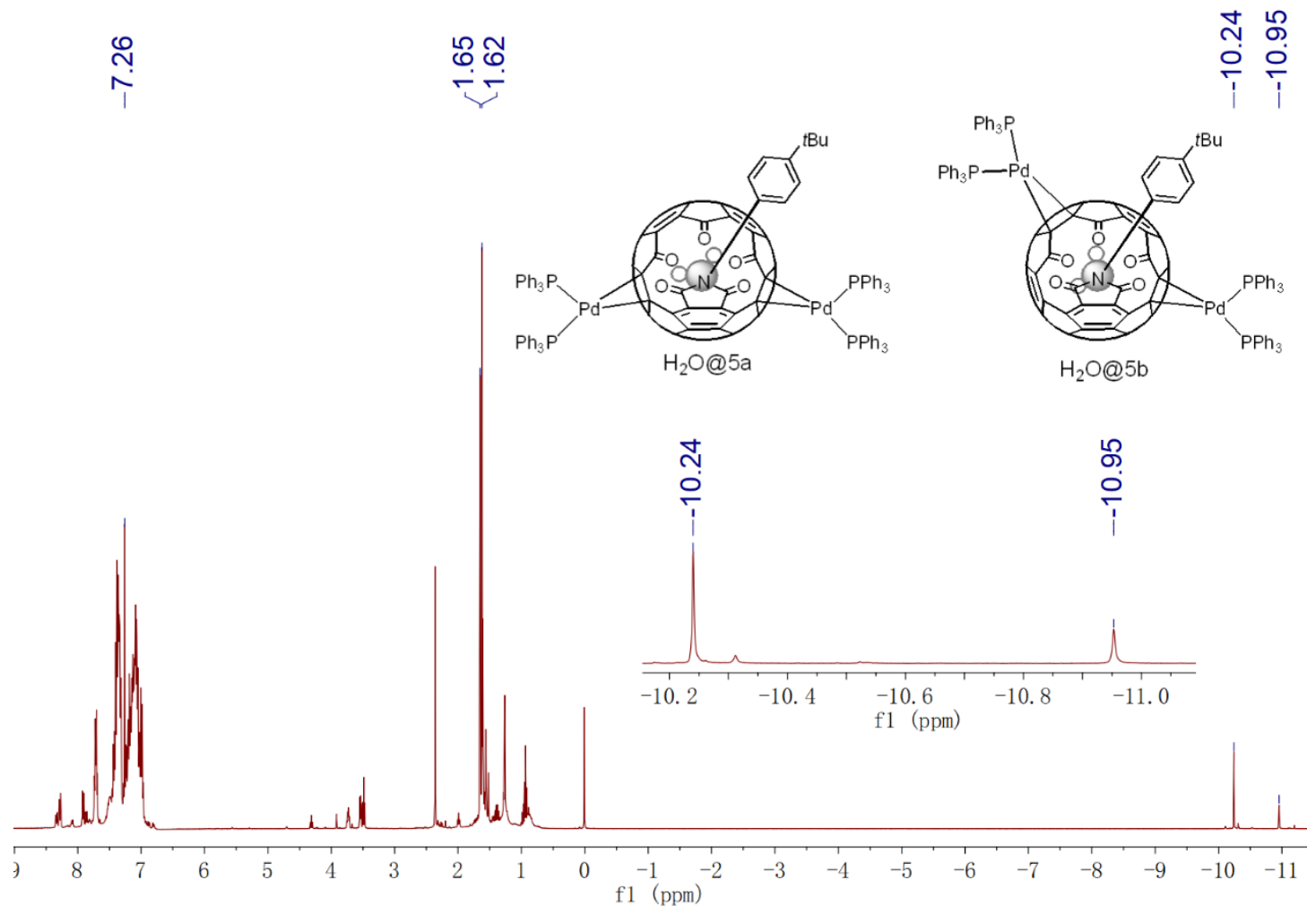


Figure S2 ${ }^{1} \mathrm{H}$ NMR of Compound $\mathrm{H}_{2} \mathrm{O} @ 6$ in $\mathrm{CDCl}_{3}$

$\stackrel{\leftrightarrow}{\stackrel{\leftrightarrow}{*}}$

○
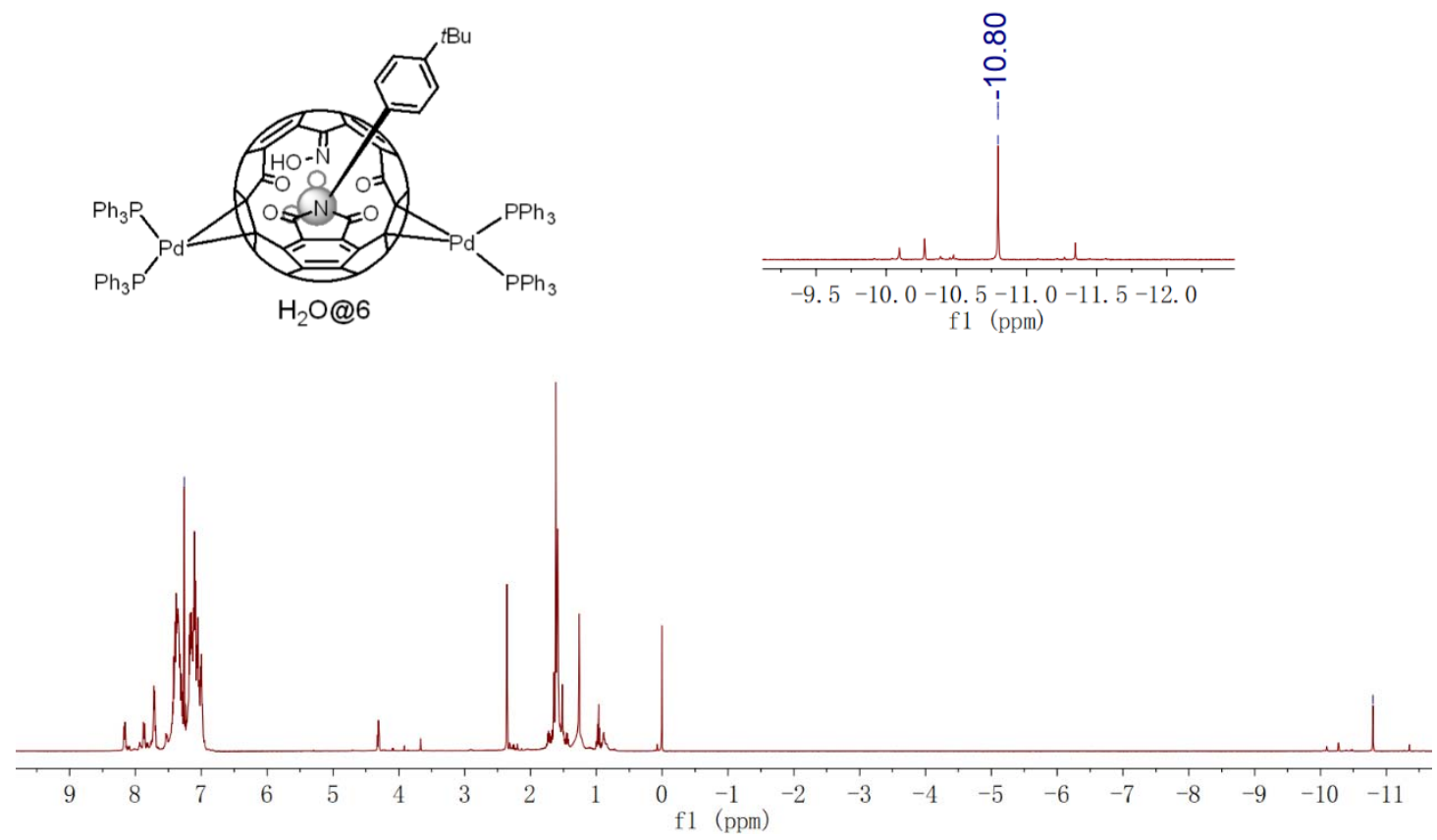

Figure S3 HRMS of Compound 4

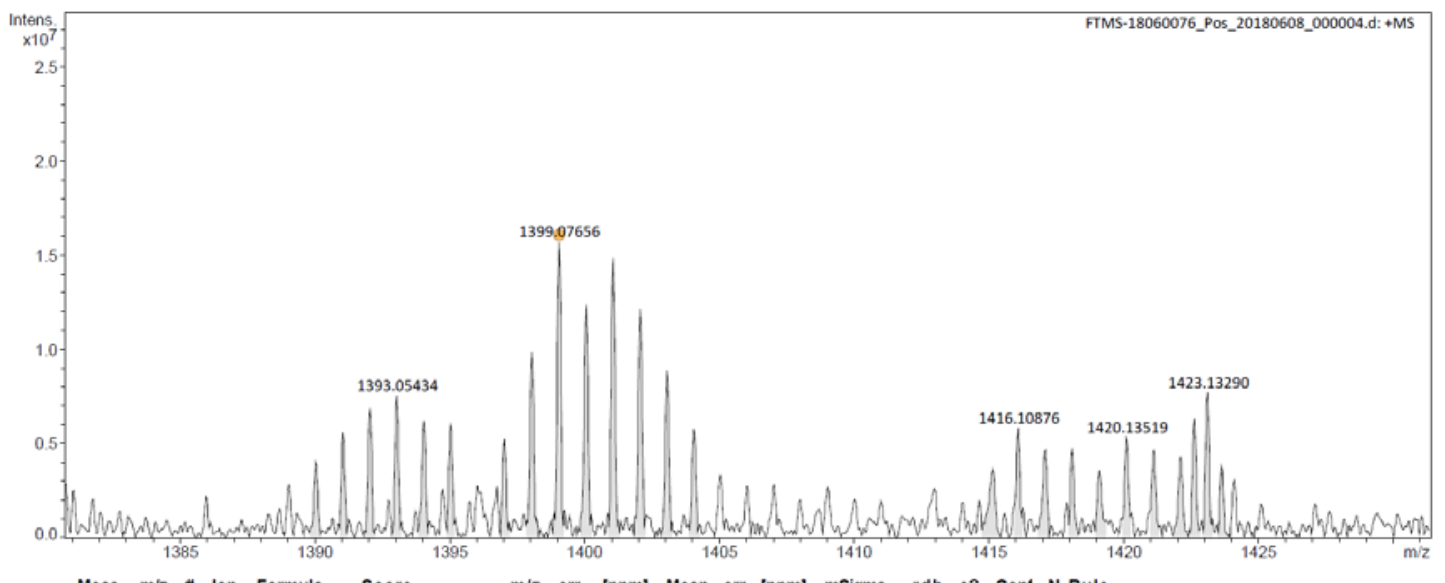

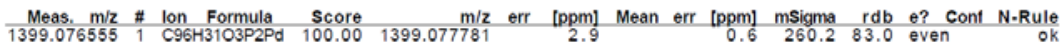


Figure S4 HRMS of Compound 5a/b

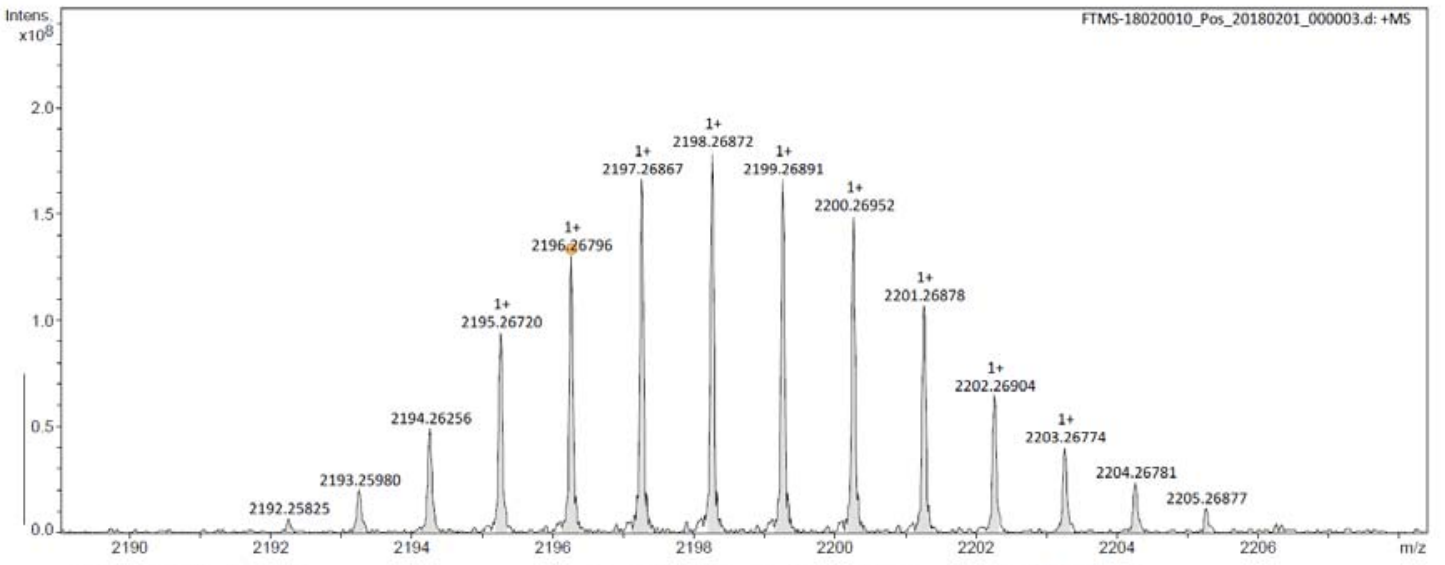

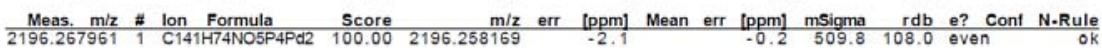

Figure S5 HRMS of Compound 6

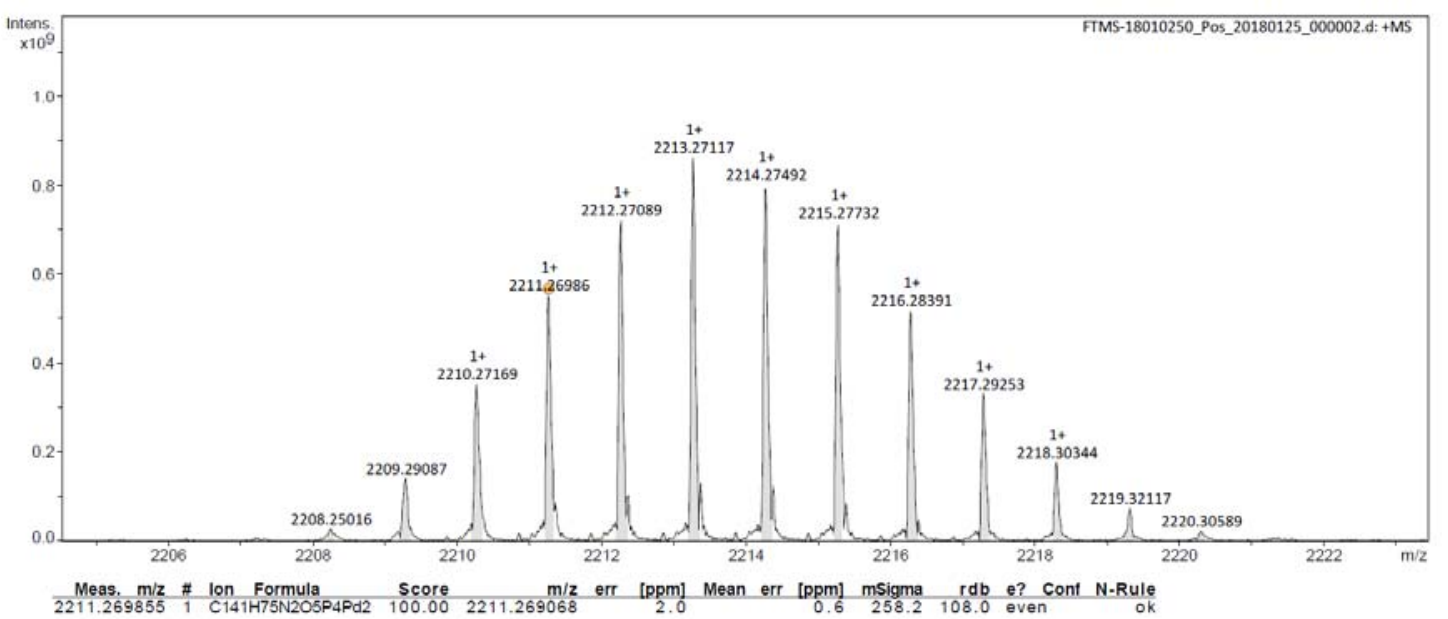


Figure S6 UV-Vis of Compounds 2, 3 \& 6 in $\mathrm{CHCl}_{3}$

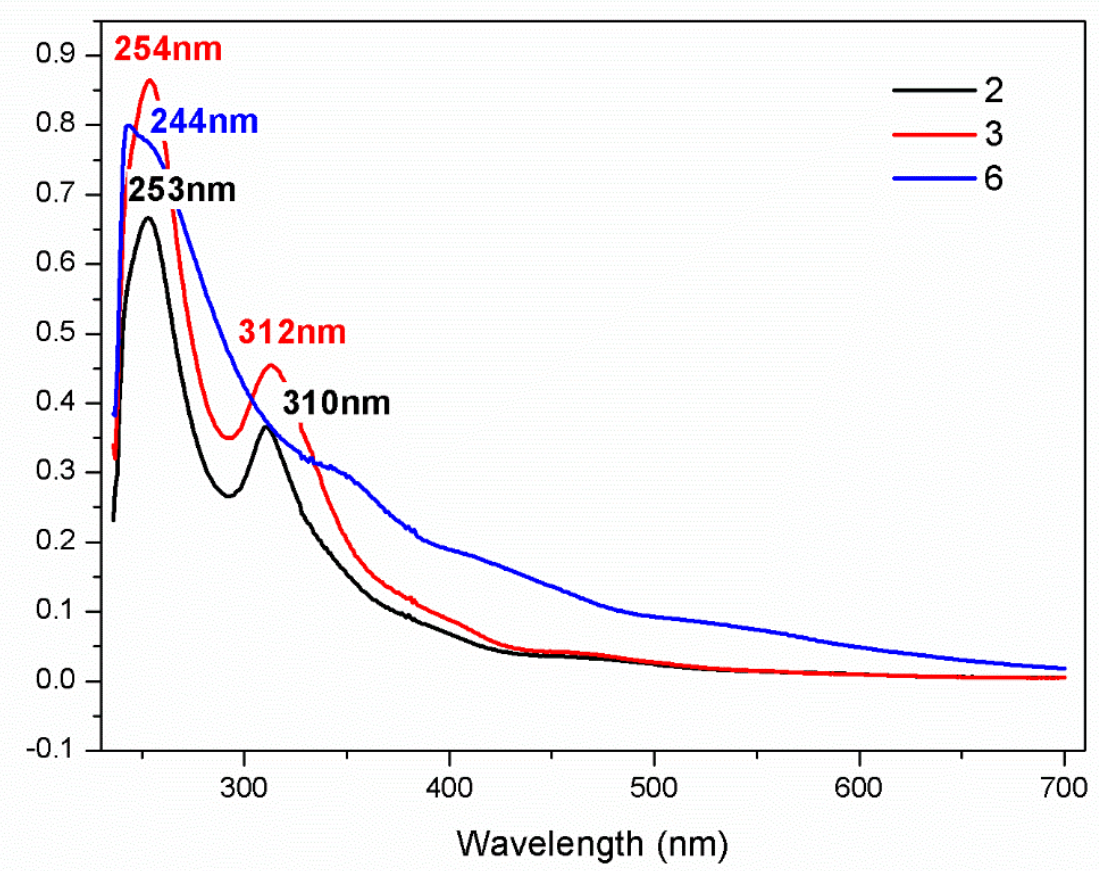

Figure S7 UV-Vis of Compounds 1, 4 in $\mathrm{CHCl}_{3}$

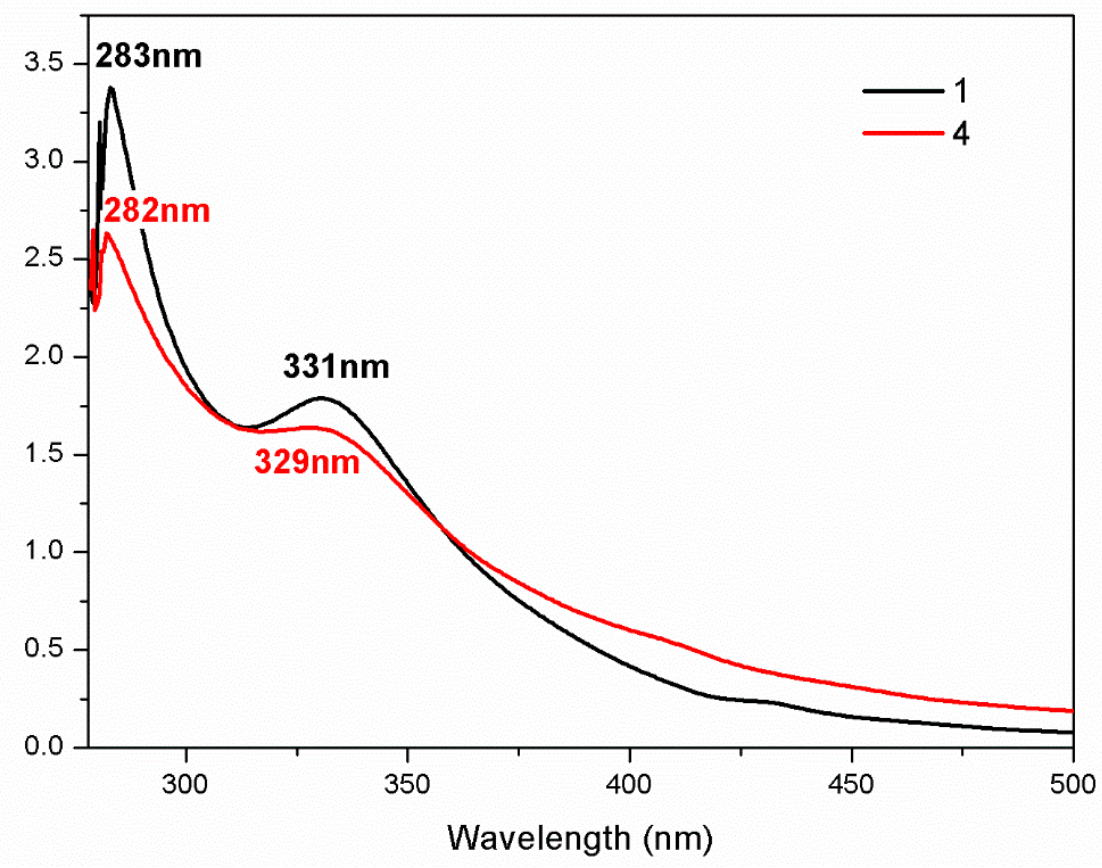




\section{Computational method}

Density functional theory (DFT) calculations were carried out by using the M06-2X functional $^{1}$ in conjunction with the Stuttgart/Dresden relativistic effective core potential and corresponding basis set ${ }^{2}$ for Pd and the standard 6-31G* basis set $^{3}$ for other elements (denoted as M06-2X/6-31G* SDD), as implemented in the Gaussian 09 software package. ${ }^{4}$ The Mayer bond orders (MBOs) were calculated by using the Multiwfn program.

From the results in Table S1 and S2, clearly, none of the component atoms of addition sites has the largest LUMO distribution. Therefore, the reactions unlikely occur by first attacking one carbon atom.
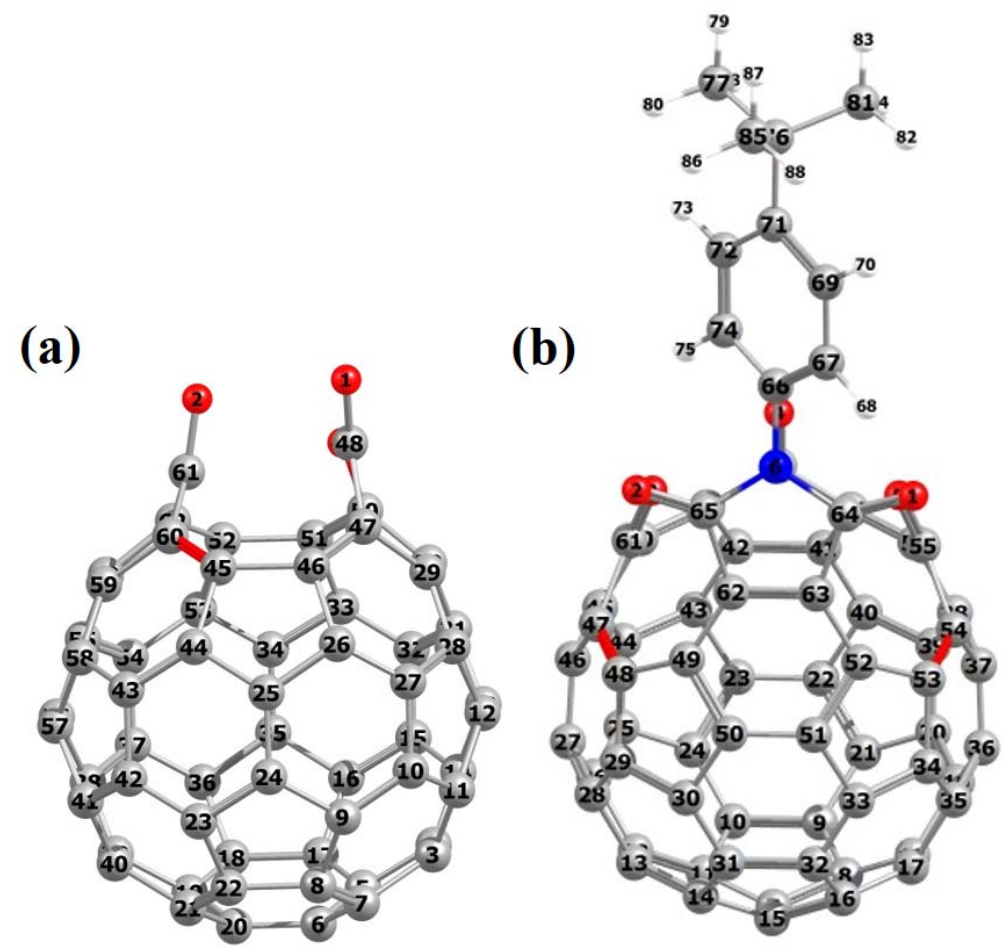

Figure S8. Optimized structures of (a) 1 and (b) 2 with the addition bonds highlighted in red.

Table S1. LUMO coefficients of all the carbon atoms on the cage of $\mathbf{1}$ (please refer to Figure S1 (a) for the atom numbering; the attacked bond atoms are shown in bold).

\begin{tabular}{cccc}
\hline Site & $\begin{array}{c}\text { LUMO } \\
(\%)\end{array}$ & Site & $\begin{array}{c}\text { LUMO } \\
(\%)\end{array}$ \\
\hline 25 & $5.97 \%$ & 36 & $1.33 \%$ \\
28 & $4.80 \%$ & 33 & $1.31 \%$ \\
58 & $4.42 \%$ & 30 & $1.00 \%$ \\
35 & $4.37 \%$ & 26 & $0.89 \%$ \\
34 & $4.25 \%$ & 16 & $0.82 \%$ \\
14 & $4.05 \%$ & 37 & $0.80 \%$ \\
24 & $3.99 \%$ & 54 & $0.77 \%$
\end{tabular}




\begin{tabular}{cccc}
\hline 46 & $3.86 \%$ & 61 & $0.70 \%$ \\
$\mathbf{4 5}$ & $\mathbf{3 . 8 0} \%$ & 53 & $0.70 \%$ \\
38 & $3.68 \%$ & 5 & $0.67 \%$ \\
4 & $2.96 \%$ & 13 & $0.66 \%$ \\
39 & $2.96 \%$ & 44 & $0.61 \%$ \\
31 & $2.71 \%$ & 63 & $0.61 \%$ \\
55 & $2.47 \%$ & 8 & $0.59 \%$ \\
41 & $2.31 \%$ & 51 & $0.56 \%$ \\
17 & $2.21 \%$ & 15 & $0.56 \%$ \\
59 & $2.21 \%$ & 22 & $0.50 \%$ \\
11 & $2.10 \%$ & 56 & $0.46 \%$ \\
29 & $2.04 \%$ & 19 & $0.45 \%$ \\
18 & $1.92 \%$ & 43 & $0.42 \%$ \\
42 & $1.90 \%$ & 57 & $0.34 \%$ \\
10 & $1.75 \%$ & 12 & $0.34 \%$ \\
52 & $1.62 \%$ & 48 & $0.33 \%$ \\
$\mathbf{6 0}$ & $\mathbf{1 . 6 0 \%}$ & 27 & $0.32 \%$ \\
9 & $1.59 \%$ & 7 & $0.21 \%$ \\
32 & $1.57 \%$ & 21 & $0.14 \%$ \\
47 & $1.41 \%$ & 20 & $0.11 \%$ \\
23 & $1.39 \%$ & 50 & $0.09 \%$ \\
40 & $1.38 \%$ & 6 & $0.07 \%$ \\
3 & $1.36 \%$ & 62 & $0.04 \%$ \\
\hline & & & \\
\hline & & 6 & \\
\hline
\end{tabular}

Table S2. LUMO coefficients of all the carbon atoms on the cage of $\mathbf{2}$ (please refer to Figure S1 (b) for the atom numbering; the attacked bond atoms are shown in bold).

\begin{tabular}{llcl}
\hline Site & $\begin{array}{c}\text { LUMO } \\
(\%)\end{array}$ & Site & $\begin{array}{c}\text { LUMO } \\
(\%)\end{array}$ \\
\hline 49 & $6.53 \%$ & 7 & $0.96 \%$ \\
52 & $6.53 \%$ & 56 & $0.82 \%$ \\
$\mathbf{4 8}$ & $\mathbf{4 . 6 6 \%}$ & 60 & $0.81 \%$ \\
$\mathbf{5 3}$ & $\mathbf{4 . 5 5 \%}$ & 50 & $0.68 \%$ \\
30 & $3.97 \%$ & 51 & $0.66 \%$ \\
27 & $3.93 \%$ & 44 & $0.55 \%$ \\
36 & $3.90 \%$ & 39 & $0.54 \%$ \\
33 & $3.90 \%$ & 34 & $0.44 \%$ \\
$\mathbf{4 7}$ & $3.47 \%$ & 29 & $0.43 \%$ \\
$\mathbf{5 4}$ & $3.41 \%$ & 14 & $0.38 \%$ \\
37 & $3.09 \%$ & 16 & $0.36 \%$ \\
46 & $3.06 \%$ & 22 & $0.32 \%$ \\
62 & $2.96 \%$ & 65 & $0.31 \%$ \\
63 & $2.94 \%$ & 23 & $0.31 \%$ \\
24 & $2.92 \%$ & 64 & $0.28 \%$ \\
21 & $2.91 \%$ & 12 & $0.24 \%$
\end{tabular}




\begin{tabular}{cccc}
\hline 20 & $2.49 \%$ & 18 & $0.23 \%$ \\
25 & $2.48 \%$ & 61 & $0.22 \%$ \\
31 & $2.37 \%$ & 55 & $0.20 \%$ \\
32 & $2.34 \%$ & 35 & $0.16 \%$ \\
10 & $1.94 \%$ & 28 & $0.16 \%$ \\
13 & $1.93 \%$ & 48 & $0.11 \%$ \\
17 & $1.90 \%$ & 38 & $0.11 \%$ \\
9 & $1.90 \%$ & 11 & $0.11 \%$ \\
19 & $1.60 \%$ & 8 & $0.10 \%$ \\
26 & $1.59 \%$ & 59 & $0.06 \%$ \\
41 & $1.37 \%$ & 15 & $0.06 \%$ \\
42 & $1.34 \%$ & 57 & $0.05 \%$ \\
40 & $1.15 \%$ & 58 & $0.03 \%$ \\
43 & $1.12 \%$ & & \\
\hline
\end{tabular}

\section{Optimized Cartesian coordinates of 1 and 2. (see separate file)}

Reference

(1) Zhao, Y.; Truhlar, D. G. The M06 suite of density functionals for main group thermochemistry, thermochemical kinetics, noncovalent interactions, excited states, and transition elements: two new functionals and systematic testing of four M06-class functionals and 12 other functionals, Theor. Chem. Acc. 2008, 120, 215-241.

(2) Andrae, D.; Häußermann, U.; Dolg, M.; Stoll, H.; Preuß, H. Energy-adjusted ab initio pseudopotentials for the second and third row transition elements, Theor. Chim. Acta 1990, 77, 123-141.

(3) Hehre, W. J.; Ditchfield, R.; Pople, J. A. Self-Consistent Molecular Orbital Methods. XII. Further Extensions of Gaussian-Type Basis Sets for Use in Molecular Orbital Studies of Organic Molecules. J. Chem. Phys. 1972, 56, 2257.

(4) Frisch, M. J.; Trucks, G. W.; Schlegel, H. B.; Scuseria, G. E.; Robb, M. A.; Cheeseman, J. R.; Scalmani, G.; Barone, V.; Mennucci, B.; Petersson, G. A.; Nakatsuji, H.; Caricato, M.; Li, X.; Hratchian, H. P.; Izmaylov, A. F.; Bloino, J.; Zheng, G.; Sonnenberg, J. L.; Hada, M.; Ehara, M.; Toyota, K.; Fukuda, R.; Hasegawa, J.; Ishida, M.; Nakajima, T.; Honda, Y.; Kitao, O.; Nakai, H.; Vreven, T.; Montgomery Jr., J. A.; Peralta, J. E.; Ogliaro, F.; Bearpark, M. J.; Heyd, J.; Brothers, E. N.; Kudin, K. N.; Staroverov, V. N.; Kobayashi, R.; Normand, J.; Raghavachari, K.; Rendell, A. P.; Burant, J. C.; Iyengar, S. S.; Tomasi, J.; Cossi, M.; Rega, N.; Millam, N. J.; Klene, M.; Knox, J. E.; Cross, J. B.; Bakken, V.; Adamo, C.; Jaramillo, J.; Gomperts, R.; Stratmann, R. E.; Yazyev, O.; Austin, A. J.; Cammi, R.; Pomelli, C.; Ochterski, J. W.; Martin, R. L.; Morokuma, K.; Zakrzewski, V. G.; Voth, G. A.; Salvador, P.; Dannenberg, J. J.; Dapprich, S.; Daniels, A. D.; Farkas, Ö.; Foresman, J. B.; Ortiz, J. V.; Cioslowski, J.; Fox, D. J. Gaussian 09; revision D.01; Gaussian, Inc.: Wallingford, CT, USA, 2013.

(5) Lu, T.; Chen, F. Multiwfn: A multifunctional wavefunction analyzer, J. Comput. Chem. 2012, 33, 580-592. 